DOI: $10.35643 /$ Info.25.2.6

Artículo original

\title{
Participación de bibliotecólogos en la edición científica en Uruguay
}

\author{
Participação dos bibliotecários na edição científica no Uruguai ${ }^{1}$ \\ Participation of librarians in scientific publishing in Uruguay
}

\begin{abstract}
Ana Laura Gilmet ${ }^{\mathrm{a}}$
a Estudiante avanzada de la Licenciatura en Bibliotecología (Facultad de Información y Comunicación, Universidad de la República, Uruguay). Correo electrónico: anagilmet@gmail.com. ORCID: 0000-0002-2160-4132.
\end{abstract}

\section{Resumen}

El presente artículo explora el trabajo de los bibliotecólogos en revistas científicas, específicamente indaga sobre las competencias, conocimientos y características de los profesionales que colaboran en la edición científica en Uruguay. Se aplica una metodología mixta: por un lado, un cuestionario a los editores de las revistas que integran la Asociación Uruguaya de Revistas Académicas, para conocer la incidencia del trabajo de los bibliotecólogos en estas revistas; por otra parte, se realizan cinco entrevistas a bibliotecólogos que trabajan en alguna de esas publicaciones para profundizar en los procesos laborales y de formación que los han llevado a participar en la edición de una revista científica. Se constata un alto porcentaje de revistas en las que trabajan bibliotecólogos, más del $40 \%$. El trabajo que realizan es muy variado en cada caso. A través de las entrevistas se profundiza en las competencias que exige la edición académica, la formación y experiencia que requiere, y el grado de integración que tienen en los equipos editores, de quienes depende en gran medida su participación.

Palabras clave: REVISTAS CIENTÍFICAS; REVISTAS ACADÉMICAS; EDICIÓN CIENTÍFICA; PROFESIONAL DE LA INFORMACIÓN; ROL DEL BIBLIOTECÓLOGO.

\section{Resumo}

Este artigo explora o trabalho dos bibliotecários nas revistas científicas, especificamente indaga a capacidade, conhecimentos e as características dos profissionais que colaboram na edição científica no Uruguai. É aplicada uma metodologia mista, por um lado um questionário aos editores das revistas que compõem a Asociación Uruguaya de Revistas Académicas, para conhecer a incidência do trabalho dos bibliotecários nessas revistas; e por outro lado, são realizadas cinco entrevistas a bibliotecários que trabalham em alguma dessas publicações com o fim de aprofundar nos processos de trabalho e de formação, 
que os levaram a participar da edição de uma revista científica. É verificada uma alta porcentagem de revistas em que trabalham bibliotecários, mais de $40 \%$. O trabalho que realizam é muito variado em cada caso. Através das entrevistas se aprofunda nas competências que exigem a edição acadêmica, a formação e experiência que ela requer; e o grau de integração que tem nas equipes editoras, das quais sua participação depende amplamente.

Palavras-chave: REVISTAS CIENTÍFICAS; REVISTAS ACADÉMICAS; EDIÇÃO CIENTÍFICA; PROFISSIONAL DA INFORMAÇÃO; ROL DO BIBLIOTECÁRIO.

\begin{abstract}
This article explores the work of librarians in scientific journals, specifically inquiring about the skills, knowledge and characteristics of professionals who collaborate in scientific publishing in Uruguay. A mixed methodology is applied: on the one hand, a questionnaire is applied to the editors of the journals that make up the Uruguayan Association of Academic Journals, to know the incidence of the work of librarians in these journals; On the other hand, five interviews are carried out with librarians who work in any of these publications to delve into the work and training processes that have led them to participate in the edition of a scientific journal. There is a high percentage of journals in which librarians work, more than $40 \%$. The work they do is very varied in each case. Through the interviews it delves into the competences, the training and experience required by academic publishing, and the degree of integration they have in the publishing teams, on whom their participation depends to a great extent.
\end{abstract}

Keywords: SCIENTIFIC JOURNALS; ACADEMIC JOURNALS; SCIENTIFIC EDITION; INFORMATION PROFESSIONAL.

Fecha de recibido: $\quad 29 / 04 / 2020$

Fecha de aceptado: $\quad 03 / 08 / 2020$

\title{
1. Introducción
}

En palabras de Andreas Würgler, citado por Capurro (2016), la revista científica es una «publicación periódica con un foco temático, una alta exigencia de calidad y de cultura de discusión crítica, que vive en base a que lectores y lectoras sean autores potenciales de la misma» (p.7). Las revistas científicas surgen en Europa en el siglo XVII como medio efectivo de comunicación de una nueva forma de hacer ciencia, que surge en los siglos anteriores, a partir del Renacimiento, sus principales características fueron la adopción del método científico, el desarrollo de contenidos originales y la certificación de estos a través de la evaluación por pares. Las revistas funcionan desde sus inicios como medio de comunicación para 
las comunidades científicas y como banco de conocimientos certificados de autoría registrada. En los siglos XVIII y XIX se profundizó la especialización de la ciencia, lo que multiplicó el número de sociedades académicas y de revistas. Pero el mayor punto de crecimiento de las publicaciones científicas tuvo lugar luego de la segunda guerra mundial, a mitad del siglo XX, con la creación de nuevas áreas del conocimiento (Borrego, 2017).

A fines del siglo XX y comienzos del siglo XXI, cambian radicalmente las estructuras de producción del conocimiento y de la comunicación científica, las revistas científicas son el medio de difusión que más cambios sufre. El actual modelo de revistas electrónicas se ha consolidado a nivel mundial luego de tres décadas de sus inicios y la publicación en papel propia de los siglos anteriores, con su sistema de distribución, dio paso al desarrollo de revistas electrónicas disponibles en la web con restricciones en su acceso o de acceso abierto (Dinu y Baiget, 2019).

El entorno digital ha habilitado el acceso abierto de las publicaciones científicas y logra constituirse en una alternativa viable a la publicación por suscripción, que venía siendo criticada desde el siglo pasado por sus altos costos. No obstante estas transformaciones, las revistas continúan siendo archivo de contenidos científicos y herramientas de reconocimiento profesional para los científicos, en tanto publicaciones arbitradas (Abadal, 2017; Sanz Menéndez, 2014).

En la actualidad, el proceso editorial comienza con la recepción de los artículos, el artículo es «etapa final de socialización del conocimiento científico» (Piezzi, 2011, p.43); en ocasiones existe un paso previo: los llamados o convocatorias para la presentación de originales. Una vez recibido un artículo es leído por el comité editorial a fin de controlar que el tema del trabajo sea adecuado para la revista, que cumpla con sus políticas, también puede realizarse una primera lectura de estilo. Una vez que el editor o editora considera que el trabajo es adecuado, se lo envía a evaluadores externos, expertos en el tema del artículo. Algunas revistas cuentan con una base de datos de evaluadores para agilizar la elección. El intercambio entre autores y evaluadores en general es anónimo (evaluación doble ciego) y siempre está mediado por el comité editorial. El editor o editora es quien decide finalmente (tomando en cuenta las recomendaciones de los evaluadores) si el trabajo se publica o no, y es el garante de la calidad del trabajo y de la 
evaluación (Jana, 2019). Por último, se realiza la corrección de estilo definitiva y se publica (Abad, 2017). El proceso no termina allí, la revista debe procurar dar visibilidad a los trabajos que publica, ya que el sentido de la publicación es que el trabajo sea conocido y discutido en la comunidad científica (Dalmagro, 2007).

En América Latina la mayoría de las revistas académicas son editadas por universidades, con fondos públicos. Es también la región con mayor acceso abierto a sus contenidos científicos; a través de bases de datos y repositorios regionales se promueve la edición de calidad y una evaluación de la ciencia alternativa a la impuesta por países desarrollados, que benefician a las grandes editoriales comerciales (Babini, 2019; Larivière et al., 2015). Uruguay se ha unido muy tarde a las iniciativas regionales que promueven el acceso abierto y no ha desarrollado políticas análogas a sus pares del sur. «Casi diez años después siguen sin existir políticas explícitas de acceso abierto de alcance nacional y los sistemas de evaluación de los investigadores siguen privilegiando la publicación en revistas de corriente principal» (Aguirre-Ligüera et al., 2019).

Si bien desde el Sistema Nacional de Investigadores (SNI) se ha afirmado que a la hora de evaluar a un investigador tiene el mismo peso un artículo publicado en una revista nacional que en una internacional (Guerra, 2018), la falta de incentivos y financiación de las revistas nacionales las deja en una posición comprometida en el momento de competir con revistas internacionales de alto impacto.

La profesionalización de los equipos editores es clave para editar revistas de calidad (Rodríguez Yunta y Tejada Artigas, 2013). En este sentido, el bibliotecólogo en una revista puede ser un actor relevante para el cumplimiento de los parámetros de calidad exigidos en las bases de datos para conseguir integrarlas y lograr visibilidad (Rozemblum y Banzato, 2009). A partir del movimiento de acceso abierto se promueve la ciencia abierta, que pone a disposición una variedad de objetos digitales además del texto del artículo científico con sus metadatos, gráficas, tablas, datos de investigación, preprints, adelantos de investigación (Babini, 2019); la normalización de estos objetos digitales extiende y complejiza las tareas que el bibliotecólogo puede realizar en una revista académica. Se considera, entonces, que la edición de revistas científicas electrónicas constituye un espacio de oportunidad laboral y profesional para el bibliotecólogo, al que le exige nuevas competencias (Santa Anna, 2019; Santana y 
Francelin, 2016). La figura del editor técnico, «rol que aglutina las nuevas funciones que conlleva la edición digital» (Rodríguez Yunta y Tejada Artigas, 2013, p.3), puede asociarse al rol del bibliotecólogo en una revista científica, pero implica competencias de gestión editorial además de las competencias tradicionales de normalización de los recursos. Aun sin desempeñar un rol próximo a un editor, el entorno actual de la edición científica exige al bibliotecólogo mantenerse activo e incorporar nuevas competencias; debe adquirir incluso conocimientos de otras profesiones, herramientas informáticas, comunicacionales y de gestión, pero también debe ser crítico y conocer en profundidad la institución a la que pertenece (Baiget, 2010). Se ha apuntado que las nuevas competencias que requiere el profesional de la información para trabajar en una revista académica deben adquirirse en la formación de grado, a través de la incorporación de estos conocimientos en los planes de estudio (Díaz Álvarez y Sánchez Terragó, 2006; Maimone y Tálamo, 2008). Sin embargo, aun cuando los planes de estudio de bibliotecología incorporen contenidos de edición científica, siempre será necesario integrar estas herramientas en la práctica laboral, según Baiget (2010), «el profesional de la información necesita poca teoría y mucha práctica» (p.37), en línea con esto, Schön afirma en El Profesional Reflexivo: «Incluso si el conocimiento profesional estuviera a la altura de las nuevas demandas de la práctica profesional, la mejora en la actuación profesional sería transitoria. Las situaciones de la práctica son, por su naturaleza, inestables» (1998, pp.25-26).

En Uruguay, la especialización en edición científica de los bibliotecólogos se da desde la práctica, a través de cursos e interacción entre colegas, cada vez de forma más sistematizada desde la creación de la Asociación Uruguaya de Revistas Académicas (AURA). Un trabajo de Valenzuela y Morelli (2018) contabiliza 58 bibliotecólogos que trabajan en la edición científica en 2015, muestra también el crecimiento de las revistas uruguayas en relación a la indización en bases de datos regionales y la sistematización del trabajo de formación de editores y bibliotecólogos a través de cursos, que tienen por objetivo aportar a la profesionalización de los equipos editores. Al día de hoy la asociación es referente en edición de revistas académicas y asesoramiento en herramientas de parámetros de calidad de las publicaciones. 
Al igual que en toda América Latina, en Uruguay las universidades son la principal institución editora de contenidos académicos, en particular la Udelar (Aguirre-Ligüera et al., 2019; Valenzuela y Morelli, 2018). Sin embargo, esta institución no tiene una política central de promoción de sus publicaciones, financiadas en su mayoría a través de dedicaciones totales de sus editores, en general docentes con grados altos. Existe una enorme diferencia en calidad, circulación y profesionalización de los equipos editores entre las revistas que editan las diferentes facultades de la Udelar, incluso en revistas editadas en una misma facultad. Ante esta realidad, y para promover sus publicaciones, la universidad está proyectando la creación de un portal universitario de revistas arbitradas, como existe en otros países de la región. Se espera que esta iniciativa venga acompañada de políticas de apoyo y financiación para las revistas que lo integren.

Una experiencia interesante para pensar el peso de las políticas institucionales que apuntan a la calidad de las revistas que edita es la relatada por Rozemblum y Banzato (2012) en la Facultad de Humanidades y Ciencias de la Educación de la Universidad Nacional de La Plata, Argentina. En este caso, trabajaron en forma coordinada los editores y bibliotecarios con el apoyo de la institución, lo que impactó de forma positiva en el desarrollo de las revistas. Destacan de esta experiencia el aporte de los bibliotecarios, no solo como mediadores de la información, sino también como agentes activos en instancias de participación. En el caso del proyecto de la Udelar, al menos en los inicios ha integrado a bibliotecólogos que trabajan en revistas académicas en las primeras instancias de participación.

Partiendo de estos antecedentes, este trabajo se propone explorar la labor de los profesionales, el objetivo es conocer las competencias, los conocimientos y las características que poseen los bibliotecólogos que colaboran en la edición de revistas científicas en Uruguay. Para esto se intenta responder a cinco objetivos específicos: 1) Explorar la incidencia que tiene la figura del bibliotecólogo en la edición científica de revistas. 2) Identificar aptitudes y características personales. 3) Reconocer conocimientos y competencias. 4) Conocer el trabajo que realizan. 5) Indagar acerca de las concepciones que tienen acerca de su profesión en el marco de la edición científica. 


\section{Metodología}

Se parte de una revisión bibliográfica a fin de contextualizar a nivel regional e internacional los temas de interés para el presente trabajo: el rol del profesional de la información en la edición académica, la actualidad de las revistas electrónicas y las competencias de los bibliotecólogos en la actualidad. Las búsquedas fueron realizadas el 10 de enero de 2020 en BiUR, Timbó, Google Scholar, Colibrí, eLIS, con los términos edición científica, bibliotecólogo, profesional de la información, rol profesional, revistas académicas, revistas científicas, editor técnico y documentalista.

Se indaga la realidad de la colaboración de los bibliotecólogos en la edición científica en el país a través de una técnica mixta, cuantitativa y cualitativa (cuestionario y entrevista respectivamente). Por un lado, se emplea un breve cuestionario que intenta responder a los objetivos 1 y 4 , aplicado a los editores de las revistas integrantes de la AURA. Se toma como referencia el listado de revistas socias de AURA publicado en la página web oficial de la asociación. El cuestionario es enviado a un grupo de correo electrónico de la red de editores ${ }^{2}$. También es enviado personalmente a los editores de las revistas que no contestaron las dos invitaciones enviadas al grupo. Se genera un listado actualizado de revistas socias de AURA, al comprobar que al menos una revista que integra el listado de referencia original no continúa publicándose y que varias revistas cuyos editores completan el cuestionario no integran el listado en la página de la asociación. Se agregan al listado varias revistas de Humanidades socias de AURA, relevadas para un trabajo anterior ${ }^{\frac{3}{}}$. El listado queda conformado por 60 revistas, se obtienen datos de 41 de ellas, 19 responden el cuestionario, el resto fueron relevadas anteriormente o se extrae la información a partir de las entrevistas. El cuestionario indaga sobre las tareas que realizan los bibliotecólogos y si forman parte del equipo estable de la revista.

Cabe aclarar que no se obtiene el número de bibliotecólogos que trabajan en revistas académicas, la incidencia de su trabajo es indagada a través del número de revistas que cuentan con su colaboración.

Por otro lado se emplea una metodología cualitativa a través de cinco entrevistas a bibliotecólogos y bibliotecólogas (una de ellas se encuentra en etapa de egreso de la Licenciatura en Bibliotecología en el momento de ser entrevistada), que intenta 
responder a los objetivos 2, 3, 4 y 5 . Se contacta a siete profesionales que participan de la edición de revistas académicas mediante la recomendación de referentes en el área de la edición, a través del contacto de los primeros entrevistados, también a partir de búsquedas directas en los datos sobre los equipos editoriales de algunas revistas. En principio, se escogen profesionales que trabajan en instituciones diferentes, de este modo, si bien no se intenta construir una muestra representativa de todos los bibliotecólogos que trabajan en revistas en Uruguay, se busca obtener diferentes experiencias. Cinco de los siete profesionales contactados acceden a ser entrevistados, todos colaboran con la edición de revistas académicas en universidades; esto reduce en parte la diversidad en el tipo de institución en la que trabajan. Sin embargo, esta circunstancia permitió conocer las diferencias entre universidades y entre diferentes servicios de la misma universidad.

Los cuadros 1 y 2 exponen las preguntas del cuestionario y de las entrevistas, y su correspondencia con los objetivos del trabajo.

\section{Cuadro 1: Cuestionario}

\begin{tabular}{|l|l|}
\hline \multicolumn{1}{|c|}{ Objetivos } & \multicolumn{1}{c|}{ Preguntas } \\
\hline $\begin{array}{l}\text { Conocer qué incidencia tiene la figura del } \\
\text { bibliotecólogo en la edición científica de } \\
\text { revistas }\end{array}$ & $\begin{array}{l}\text { ¿El bibliotecólogo/a forma parte del equipo } \\
\text { editorial o trabaja desde fuera? }\end{array}$ \\
\hline Conocer el trabajo que realizan & ¿Qué tareas realiza? \\
\hline
\end{tabular}

Fuente: elaboración propia

Cuadro 2: Entrevista

\begin{tabular}{|c|c|}
\hline Objetivos & Preguntas \\
\hline $\begin{array}{l}\text { Identificar aptitudes y características } \\
\text { personales }\end{array}$ & $\begin{array}{l}\text { ¿Se integró al equipo editorial a través de } \\
\text { una demanda o su carácter proactivo los } \\
\text { llevó a recomendar su colaboración? }\end{array}$ \\
\hline Conocer el trabajo que realizan & $\begin{array}{l}\text { ¿Qué tareas realiza? } \\
\text { ¿Ha variado su participación desde su } \\
\text { ingreso hasta hoy? }\end{array}$ \\
\hline Identificar conocimientos y competencias & $\begin{array}{l}\text { ¿Qué conocimientos debió poner en juego } \\
\text { para realizar estas tareas y de qué forma los } \\
\text { ha adquirido? } \\
\text { Además de las competencias tradicionales y } \\
\text { los nuevos conocimientos tecnológicos, los } \\
\text { bibliotecólogos que trabajan en revistas } \\
\text { ¿tienen una mirada crítica (política y } \\
\text { epistemológica) respecto de los sistemas de } \\
\text { evaluación nacionales y las métricas de } \\
\text { evaluación de las publicaciones? ¿Deben } \\
\text { tenerla? }\end{array}$ \\
\hline
\end{tabular}


Indagar acerca de las concepciones que tienen acerca de su profesión en el marco de la edición científica
¿Cuál considera que es el aporte distintivo de un bibliotecólogo en una revista científica? ¿Qué visión tiene acerca del rol que deben desempeñar los bibliotecólogos en el ámbito de la producción científica?

Fuente: elaboración propia

\section{Resultados}

\subsection{Revistas con bibliotecólogos}

Como quedó establecido en el punto anterior, se obtienen datos de 41 revistas sobre 60 de la muestra inicial. Se constata que 26 revistas cuentan con bibliotecólogos y 15 no cuentan con ellos. Sobre 20 revistas no se obtienen datos. El gráfico 1 muestra un porcentaje importante de revistas en las que trabajan bibliotecólogos, 42,62 \% en base a las 60 revistas de la muestra.



Fuente: elaboración propia

Ese 42,62 \% (26 revistas) está constituido por 15 revistas de Ciencias Sociales y Humanidades (SCyH), 10 revistas de Ciencias, Tecnología, Ingeniería y Matemáticas (STEM por sus siglas en inglés) y una revista interdisciplinaria, como muestra el gráfico 2 .

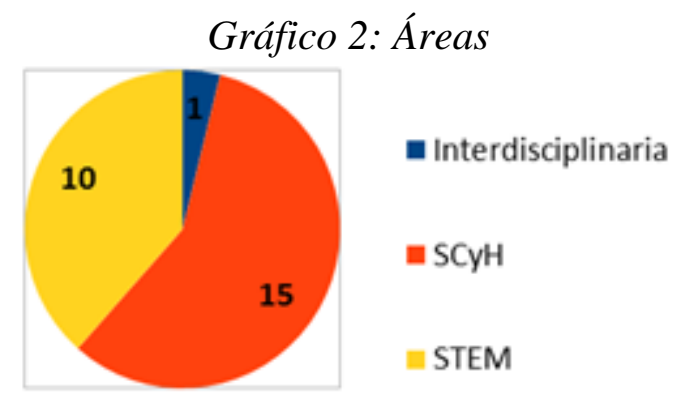

Fuente: elaboración propia 
De las 26 revistas que cuentan con bibliotecólogos, más del $60 \%$ corresponde a universidades, 9 a la Udelar, 8 a universidades privadas (5 de UCU, 2 de UM y 1 de ORT), 4 a asociaciones científicas o profesionales, 3 a instituciones educativas privadas, por último, una editorial comercial y una institución gubernamental. El gráfico 3 muestra las diferentes instituciones editoras que cuentan con bibliotecólogos y las que no.

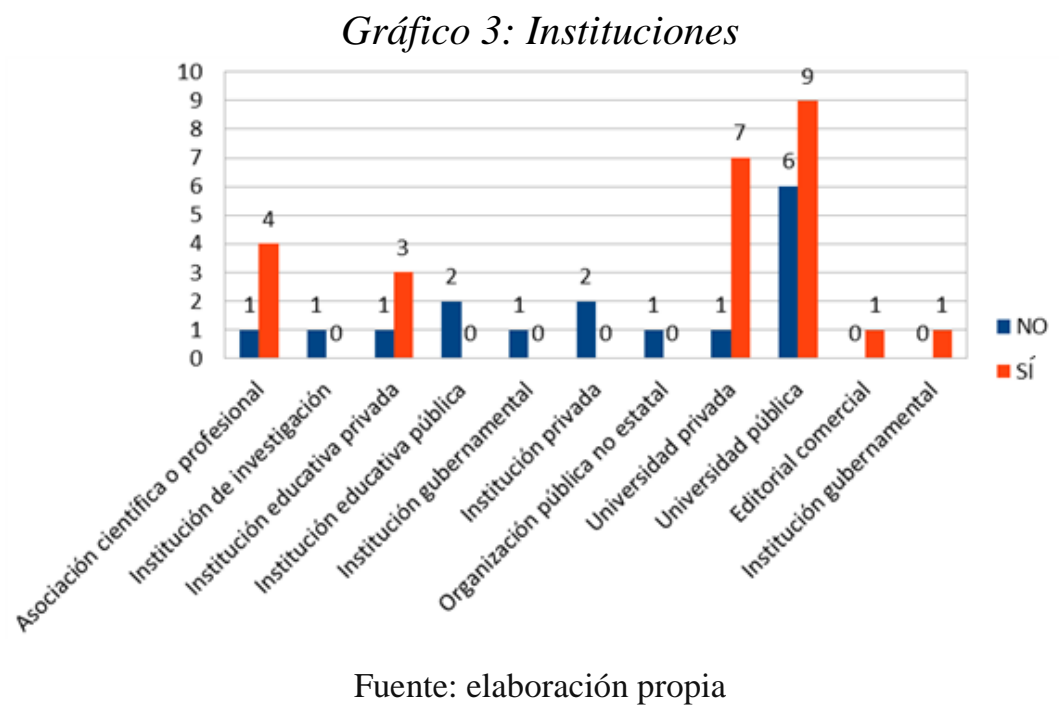

Resulta interesante observar los datos de las revistas de la Udelar que han podido ser relevados, 9 cuentan con bibliotecólogos (5 de SCyH, 4 de STEM) y 6 no cuentan con bibliotecólogos (todas de $\mathrm{SCyH}$ ). En principio, esto da cuenta de la diversidad en el funcionamiento de las revistas editadas por la misma institución. Esto también está presente en lo que concierne a las tareas de los bibliotecólogos, que van desde una colaboración puntual en el marcapeo de los artículos hasta una participación muy activa en el equipo editorial, incluso desarrollando tareas editoriales y de toma de decisiones. En el caso de la Universidad Católica del Uruguay, por ejemplo, la colaboración de los bibliotecólogos está mediada por la biblioteca y el trabajo es más sistemático, esto depende de las políticas de publicación académica de la institución, que repercute en el trabajo de los bibliotecólogos.

La totalidad de editores que respondieron el cuestionario afirman que el o los profesionales de la información que trabajan en la revista forman parte del equipo editorial. Por fuera del cuestionario se constató que en una revista trabaja una bibliotecóloga de manera puntual realizando el marcapeo de los artículos. En 
cuanto a las tareas que realizan los bibliotecólogos, agregaron al listado ofrecido en las preguntas las tareas de difusión, editor y secretaria de redacción. La cantidad de tareas que realizan los bibliotecólogos difiere de un caso a otro, desde una tarea a 13, el promedio es de 7 tareas diferentes. El gráfico 4 muestra las tareas que realizan los bibliotecólogos en orden de frecuencia.

\section{Gráfico 4: Tareas}

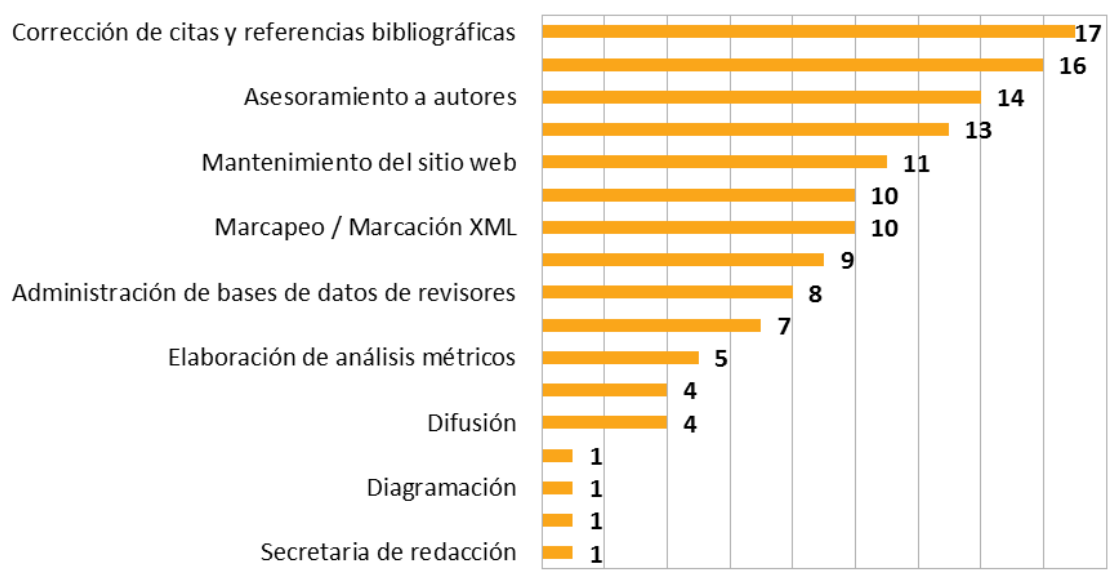

Fuente: elaboración propia

\subsection{Ingreso y tareas}

El ingreso a las revistas responde en todos los casos a una demanda de la publicación. En general, se trata de un nuevo cargo creado que los profesionales ocupan a través de un llamado o aceptando una invitación más informal, ofrecimiento basado en el conocimiento sobre la formación y competencias previas de los profesionales. Algunos de esos cargos fueron ocupados previamente por otras personas. En la mayoría de los casos, los profesionales trabajan en más de una revista, colaboran o han colaborado en dos y hasta en siete publicaciones a la vez. En general, estos poseían conocimientos previos e interés en desarrollarse en el ámbito de la edición científica.

Por lo regular, los profesionales ingresan realizando tareas tradicionales de la profesión, como el control bibliográfico de los artículos. En varios casos se agregan con el tiempo tareas de edición y gestión de herramientas informáticas, asumiendo nuevas responsabilidades delegadas por el equipo editorial, en tanto la revista o la relación laboral evoluciona. El cuadro 3 muestra las tareas 
desarrolladas por los profesionales al inicio de su incorporación a la revista y las que se agregaron después.

Cuadro 3: Tareas

\begin{tabular}{|l|l|}
\hline \multicolumn{1}{|c|}{ Tareas iniciales } & \multicolumn{1}{c|}{ Tareas adicionales } \\
\hline $\begin{array}{l}\text { Revisión y corrección bibliográfica de los } \\
\text { artículos }\end{array}$ & $\begin{array}{l}\text { Marcaje de artículos para ingreso a bases de } \\
\text { datos }\end{array}$ \\
\hline $\begin{array}{l}\text { Corrección de citas y referencias, figuras } \\
\text { y tablas }\end{array}$ & Análisis de métricas \\
\hline $\begin{array}{l}\text { Marcaje de artículos para ingreso a bases } \\
\text { de datos }\end{array}$ & $\begin{array}{l}\text { Creación y mantenimiento del portal } \\
\text { institucional de revistas académicas }\end{array}$ \\
\hline Migración de software & Gestión de ISBN e ISSN \\
\hline Gestión de OJS & Asignación de DOI y ORCID \\
\hline Diseño web & $\begin{array}{l}\text { Colaboración en otras publicaciones de la } \\
\text { institución }\end{array}$ \\
\hline Comunicación con autores y revisores & Utilización de software antiplagio \\
\hline Coordinación de eventos & Participación en redes de editores \\
\hline Editora / Editora ejecutiva & Comunicación con autores y revisores \\
\hline & $\begin{array}{l}\text { Asesoramiento sobre derecho de autor y } \\
\text { plagio }\end{array}$ \\
\hline
\end{tabular}

Fuente: elaboración propia

Las tareas iniciales que exceden la formación en bibliotecología corresponden a conocimientos y experiencias previas de los profesionales. Es importante aclarar además que varias personas entrevistadas trabajan en más de una revista y, al ingresar a una segunda, desarrollan tareas al inicio que en la revista donde empezaron fueron tareas adicionales.

\subsection{Competencias}

Este punto trata sobre las competencias necesarias para desarrollar las tareas descritas. En la mayoría de los casos el ingreso de los profesionales a las revistas se vio facilitado por conocimientos y competencias que habían adquirido previamente. Describe cuáles son esas competencias y de qué manera fueron adquiridas. Se entiende por competencias la siguiente definición:

Conjunto de capacidades necesarias para ejercer una actividad profesional y para dominar los comportamientos que se requieren. Los componentes son los siguientes: los conocimientos teóricos, los conocimientos técnicos y las aptitudes. Estos componentes deben ser operativos, aplicados en la práctica y validados (European Council of Information Associations, 2004).

Las competencias en información son las que adquiere el profesional en la formación de grado en bibliotecología. Se trata de los conocimientos adquiridos 
sobre las publicaciones, particularmente las publicaciones periódicas de contenido académico. Incluye nociones sobre la calidad y normalización técnica de las revistas, los artículos y partes ellos, el control de resúmenes, las palabras clave, las referencias bibliográficas, los descriptores. También implica conocer los parámetros de calidad que exigen las bases de datos, el entorno de la publicación. Los entrevistados lo mencionan como el «trabajo técnico», el control sobre los «parámetros técnicos» que aporta «valor agregado» a los contenidos de las publicaciones.

El perfil de graduación de la Licenciatura en Bibliotecología de la Facultad de Información y Comunicación (FIC) de la Udelar incluye en su descripción de competencias el asesoramiento en el campo editorial. Sin embargo, de las entrevistas se desprende que el aporte de la carrera de grado en cuanto a la edición científica en publicaciones periódicas es limitado. Describen el aporte del grado como un «muestrario» de contenidos sobre los que es necesario profundizar, y que no se corresponden del todo a la dinámica de publicación de una revista académica. En general esta formación se completa en cursos específicos del tema, posgrados y de forma autodidacta.

En cuanto a las competencias en tecnologías, el manejo de las TIC también se encuentra en la descripción del egresado en bibliotecología. Al respecto se señala directamente la ausencia de contenidos transmitidos en el grado, como el lenguaje de programación, base de las herramientas de marcación y etiquetado de los artículos muy presente en el desarrollo de una revista. Este tipo de conocimientos se adquiere fundamentalmente en cursos, en la práctica laboral y de manera autodidacta.

Las competencias de comunicación y gestión no son propias del perfil del bibliotecólogo. No todos los bibliotecólogos que trabajan en revistas desarrollan tareas de comunicación, los que sí lo hacen adquieren estas habilidades en la práctica laboral, al participar e incluso coordinar eventos, reuniones, etc.

Por otro lado, gestionar una revista no es una tarea propia del bibliotecólogo, no se describe en el perfil de egreso mencionado y no es el rol que cumplen en general los profesionales de la información, es un rol que desempeña un académico, por lo regular de alto grado, referente del tema de la revista. En otros 
países, en cambio, sí se habla del documentalista como editor técnico, como se mencionó en la introducción.

Una de las bibliotecólogas entrevistadas es editora responsable en una revista y editora técnica en otra. Adquirió las competencias necesarias para el puesto luego de trabajar muchos años en una editorial y a través de cursos. Otra de las personas entrevistadas cuenta con una formación técnica previa en edición.

Sobre las competencias necesarias que requiere un editor se extraen entrevistas y algunos conceptos interesantes. El editor va incorporando muchas herramientas y habilidades en la práctica editorial permanentemente. Una de las entrevistadas afirma, en línea con Schön (1998) y Baiget (2010), expuesto en la introducción, «el editor tiene que saber esto y puede hacerlo en un curso, pero después va a venir la otra parte que es de curiosidad, de emprendimiento». Es decir, la formación de editor nunca es acabada e implica siempre una aptitud de búsqueda autodidacta.

En cuanto a otros saberes, el conocimiento en otras áreas académicas, además de la información, también aporta competencias al profesional, que puede enriquecer su desempeño, dependiendo del grado de participación que tenga en la revista. Según las opiniones vertidas en las entrevistas, los profesionales consideran que el bibliotecólogo debe tener cultura general, claro que esta se obtiene por muchas vías además de la formación académica.

La capacidad de trabajar en equipo es otra habilidad destacada por los entrevistados, adquirida en experiencias laborales previas y otras actividades.

También depende en gran medida de las características personales de los profesionales. Lo mismo que sucede con el conocimiento crítico que todos consideran necesario, todos los entrevistados coinciden en que el bibliotecólogo debe tener una opinión formada en cuanto a los sistemas de evaluación, las bases de datos. La apreciación crítica del mundo de la edición científica es fundamental para asesorar a quienes toman decisiones en una revista. Se considera necesario tomar conciencia de la estructura del sistema de ciencia nacional e internacional, que es funcional «para otro mundo», no para la región en la que nos encontramos. El cuadro 4 sistematiza lo expuesto anteriormente. 


\section{Cuadro 4: Competencias}

\begin{tabular}{|c|c|c|}
\hline $\begin{array}{l}\text { Grupos de } \\
\text { competencias }\end{array}$ & Competencias & Adquisición de competencias \\
\hline \multirow{4}{*}{$\begin{array}{l}\text { Grupo I } \\
\text { Información }\end{array}$} & \multirow{4}{*}{$\begin{array}{l}\text { Conocimiento de publicaciones } \\
\text { y edición científica } \\
\text { Conocimientos específicos en } \\
\text { normas bibliográficas, } \\
\text { normalización de publicaciones }\end{array}$} & Formación académica de grado \\
\hline & & Formación académica de posgrado \\
\hline & & Cursos de actualización profesional \\
\hline & & Formación autodidacta \\
\hline \multirow{3}{*}{$\begin{array}{l}\text { Grupo T } \\
\text { Tecnologías }\end{array}$} & $\begin{array}{l}\text { Manejo de herramientas } \\
\text { informáticas, software de } \\
\text { gestión editorial }\end{array}$ & $\begin{array}{l}\text { Formación en Informática } \\
\text { Cursos } \\
\text { Práctica laboral } \\
\text { Formación autodidacta }\end{array}$ \\
\hline & $\begin{array}{l}\text { Manejo de herramientas de } \\
\text { diseño }\end{array}$ & $\begin{array}{l}\text { Formación en diseño web } \\
\text { Formación autodidacta }\end{array}$ \\
\hline & $\begin{array}{l}\text { Lenguaje HTML, marcación, } \\
\text { métricas }\end{array}$ & Cursos \\
\hline $\begin{array}{l}\text { Grupo C } \\
\text { Comunicación }\end{array}$ & Habilidades comunicativas & $\begin{array}{l}\text { Práctica laboral } \\
\text { Participación en actividades en el medio }\end{array}$ \\
\hline $\begin{array}{l}\text { Grupo G } \\
\text { Gestión }\end{array}$ & $\begin{array}{l}\text { Conocimientos de gestión } \\
\text { editorial }\end{array}$ & $\begin{array}{l}\text { Experiencia laboral previa en editorial } \\
\text { Cursos de edición } \\
\text { Formación técnica en edición } \\
\text { Formación autodidacta }\end{array}$ \\
\hline \multirow{3}{*}{$\begin{array}{l}\text { Grupo } \mathrm{S} \\
\text { Otros saberes }\end{array}$} & $\begin{array}{l}\text { Competencias académicas en } \\
\text { otras disciplinas }\end{array}$ & Formación académica en otras carreras \\
\hline & $\begin{array}{l}\text { Aptitud para el trabajo en } \\
\text { equipo }\end{array}$ & $\begin{array}{l}\text { Actividades artísticas } \\
\text { Práctica laboral en otros rubros }\end{array}$ \\
\hline & Apreciación crítica & $\begin{array}{l}\text { Conocimiento crítico de la institución y } \\
\text { del mundo de la edición científica, } \\
\text { nacional, regional e internacional } \\
\text { Participación en instancias de toma de } \\
\text { decisiones }\end{array}$ \\
\hline
\end{tabular}

Fuente: elaboración propia en base a las categorías de competencias del Euro-referencial

\subsection{Participación y rol del bibliotecólogo}

Existe una relación estrecha entre la voluntad del equipo editor (o editor en jefe) y la participación del bibliotecólogo. El equipo editorial determina en gran medida la participación del profesional, por ejemplo, al incorporarlo a las reuniones de coordinación de la revista. En una experiencia se menciona que la participación del mismo profesional en la misma revista se vio restringida luego de un cambio en el equipo editor.

En varios casos se menciona que a partir de esas reuniones, de esa participación, surgen nuevas tareas; el bibliotecólogo puede definir qué es lo que puede hacer, puede proponer y dar a conocer al equipo editorial las tareas que puede realizar, el aporte a la revista. La cooperación interdisciplinaria en el equipo editorial es mencionada en varios casos como una forma de trabajo que favorece la calidad de 
la revista. En general los entrevistados hablan de una relación fluida y de confianza con el equipo editor. La diferencia en el uso del «nosotros» y del «ellos» para referirse al equipo de la revista muestra el grado de participación en la revista, la distancia.

La participación además se da a nivel macro, por ejemplo, en la toma de decisiones políticas institucionales, como en el caso del proyecto reciente de creación de un portal universitario de revistas arbitradas de la Udelar. Tiene que ver además, como fue mencionado, con la apreciación crítica que tiene el profesional de la institución a la que pertenece. El funcionamiento institucional, coordinación, comunicación, gestión, decisiones políticas. Son conscientes no solo de la revista para la que trabajan, ubican su trabajo en estructuras mayores, en el tipo de institución.

Por otro lado se manifiesta que el grado de integración también depende del mismo profesional y de su interés en participar y abandonar el «complejo de inferioridad», la actitud «cómoda» de la «queja» por la «falta de reconocimiento» presente en la profesión. De modo que la apreciación crítica debe poner el foco también en este aspecto.

Además del trabajo técnico del bibliotecólogo, expuesto en el punto anterior sobre las competencias en información, el profesional puede aportar calidad a las revistas participando activamente, atento a la dinámica de la investigación científica: «El profesional de la información que tiene que postular, presentar a bases de datos, tener la revista adecuadamente, siguiendo todos los parámetros de calidad para postularse a esas bases, y ¿qué es lo que te da eso? y visibilidad». Para esto debe mantenerse actualizado: «El rol del bibliotecólogo debe ser sumamente activo, se deben realizar capacitaciones periódicas y mantenerse informado a través de diferentes canales en cuanto a novedades en el campo editorial».

Por último, es de interés señalar a partir de las entrevistas el rol formador que puede tener el bibliotecólogo que trabaja en una revista científica. Esto se da en una dimensión cercana de la práctica laboral, donde el profesional que adquirió competencias en un curso o de forma autodidacta replica este saber a otro. También en un plano más general, por ejemplo, a través de la organización de talleres, compartiendo herramientas aprendidas en el exterior. Esto comenzó a 
articularse de forma sistemática a partir de la creación de AURA, que tiene, entre otros, el objetivo de promover la profesionalización de los equipos editores.

\section{Consideraciones finales}

Queda claro que las competencias previas de los profesionales influyen en su ingreso a las revistas. En general, han adquirido esas competencias por fuera de sus estudios de grado en bibliotecología, en el que encuentran escasa formación sobre las publicaciones científicas y herramientas informáticas, como el lenguaje HTML. Sería recomendable, por tanto, la incorporación de estos conocimientos en la carrera. Por un lado, los propios profesionales podrían proponerlo participando de instancias de cogobierno de la FIC, a través del orden de egresados. El presente trabajo no explora la existencia de iniciativas al respecto. Por otro lado, pueden aportar a la visibilización del trabajo que realizan en el estudiantado de la facultad, como relata una de las entrevistadas, rescatando la potencia de participar en las bienvenidas de las nuevas generaciones de estudiantes de bibliotecología, y mostrar el trabajo de los profesionales en las revistas académicas.

De todas maneras, es difícil que la formación de grado incorpore de manera totalmente actualizada todas las herramientas emergentes, dado el dinamismo presente en la edición científica. Es probable que muchos conocimientos continuarán transmitiéndose en cursos puntuales o en la práctica laboral.

Otras competencias están muy emparentadas con las características personales de los profesionales, la inquietud de saber, que lo empujan a aprender de manera autodidacta; el sentido crítico, que lleva a comprender el apabullante mundo de la edición científica con una perspectiva regional.

Por último, lo investigado en el presente trabajo da cuenta de los nuevos espacios habitados por el bibliotecólogo en Uruguay, que implica un desarrollo y una ampliación del rol profesional desde sus inicios. Es importante destacar el modo participativo y colaborativo que asumen al habitar esos nuevos roles.

\section{Referencias bibliográficas}

Abad, M. F. (2017). El proceso de revisión por pares. En E. Abadal (Ed.), Revistas científicas: situación actual y retos a futuro (pp. 53-72). Edicions de la Universitat de Barcelona. 
Abadal, E. (2017). Las revistas científicas en el contexto del acceso abierto. En E. Abadal (Ed.), Revistas científicas: situación actual y retos a futuro (pp. 181-196). Edicions de la Universitat de Barcelona.

Aguirre-Ligüera, N., Maldini, J., y Fontans, E. (2019). Acceso abierto a la producción científica de Uruguay: poca historia en 10 años (2009-2018). Palabra Clave, 9(1). https://doi.org/10.24215/18539912e079

Babini, D. (2019). La comunicación científica en América Latina es abierta, colaborativa y no comercial: desafíos para las revistas. Palabra Clave, 8(2). https://doi.org/10.24215/18539912e065

Baiget, T. (2010). Profesionales de la información: un futuro de oportunidades. Recuperado de http://eprints.rclis.org/15086/1/Profesionales.pdf

Borrego, Á. (2017). La revista científica: un breve recorrido histórico. En E. Abadal (Ed.), Revistas científicas: situación actual y retos a futuro (pp. 19-34). Edicions de la Universitat de Barcelona. Recuperado de http://www.publicacions.ub.edu/ficha.aspx?cod=08719

Capurro, R. (2016). ¿Qué es una revista científica? Informatio, 20(1). Recuperado de https://informatio.fic.edu.uy/index.php/informatio/article/view/165

Dalmagro, M. C. (2007). Cuando de textos científicos se trata...: guía práctica para la comunicación de los resultados de una investigación en ciencias sociales y humanas (4. ${ }^{\mathrm{a}}$ ed.). Comunicarte: Córdoba.

Díaz Álvarez, Y. Y., y Sánchez Terragó, N. (2006). Identificación de competencias en edición para los profesionales de la información. Acimed, 14(2). Recuperado de http://eprints.rclis.org/9195/

Dinu, N.-R., y Baiget, T. (2019). Presente y futuro de las revistas científicas. Scire: Representación y Organización del Conocimientos, 25(1), 37-46.

European Council of Information Associations. (2004). Euro-referencial en información y comunicación. Madrid: SEDIC. Recuperado de https://www.sedic.es/wp-content/uploads/2019/06/euref1-espanol.pdf

Guerra, P. (2018). ¿Cómo evaluar las revistas científicas? Acerca de las limitaciones del Índice de Impacto por citas. Revista de la Facultad de Derecho, 44(ene.-jun.). Recuperado de https://revista.fder.edu.uy/index.php/rfd/article/view/598

Jana, S. (2019). A history and development of peer-review process. Annals of Library and Information Studies, 66(4), 152-162. 
Larivière, V., Haustein, S., y Mongeon, P. (2015). The oligopoly of academic publishers in the digital Era. PLOS ONE, 10(6).

https://doi.org/10.1371/journal.pone.0127502

Maimone, G., y Tálamo, M. de F. (2008). A atuação do profissional da informação no processo de editoração de periódicos científicos. Revista $A C B, 13(2), 301-321$.

Piezzi, R. S. (2011). Políticas editoriales de instituciones y editoriales: aquí y ahora de las ediciones científicas de la región. En M. S. Piccone y M. Jousset (Eds.), Impacto y visibilidad de las revistas científicas (pp. 43-49). Buenos Aires: Biblioteca Nacional. Recuperado de http://www.centroredes.org.ar/wpcontent/uploads/2019/02/Impacto_y_visibilidad_Rev_Cientif.pdf

Rodríguez Yunta, L., y Tejada Artigas, C. M. (2013). El editor técnico: un perfil necesario para la profesionalización de la edición de revistas científicas en el entorno digital. Anales de Documentación, 16(2), 1-9. https://doi.org/10.6018/analesdoc.16.2.176391

Rozemblum, C., y Banzato, G. (2009). Nuevos roles del bibliotecario en las estrategias de publicación de revistas académicas. VII Encuentro de Editores de Revistas Científicas, La Habana. http://www.memoria.fahce.unlp.edu.ar/trab_eventos/ev.522/ev.522.pdf

Rozemblum C., y Banzato, G. (2012). La cooperación entre editores y bibliotecarios como estrategia institucional para la gestión de revistas científicas. Información, Cultura y Sociedad, O(27). Recuperado de http://ppct.caicyt.gov.ar/index.php/ics/article/view/1983

Santa Anna, J. (2019). O bibliotecário na editoração de periódicos científicos eletrônicos: possibilidades empreendedoras. Informatio, 24(1), 25-41. https://doi.org/10.35643/Info.24.1.3

Santana, S. A., y Francelin, M. M. (2016). O bibliotecário e a editoração de periódicos científicos. Revista Brasileira de Biblioteconomia e Documentação, 12(1), 2-26.

Sanz Menéndez, L. (2014). La evaluación de la ciencia y la investigación. Revista Española de Sociología, 21, 137-148.

Schön, D. A. (1998). El profesional reflexivo: cómo piensan los profesionales cuando actúan. Barcelona: Paidós. 
Valenzuela, C., y Morelli, T. (2018). Profesionalización de la edición científica: una mirada empírica sobre la situación en Uruguay. 8. ${ }^{\mathrm{a}}$ Conferencia internacional sobre revistas científicas, Barranquilla. Recuperado de http://thinkepi.net/notas/crecs_2018/V9_30_Valenzuela.pdf

\section{Notas}

${ }^{1}$ La traducción del título, resumen y palabras clave en portugués fue realizada por la Lic. Lucía Kleist Machado.

${ }^{2}$ El acceso al grupo de correo electrónico de la red de editores fue facilitado muy amablemente por la editora Lic. Teresa Morelli, quien también ofreció asesoramiento, bibliografía y datos.

${ }^{3}$ Se incluyen datos relevados para el trabajo final de grado «Edición de revistas académicas de Humanidades en Uruguay: circulación, desafíos y retos a futuro» (en proceso) para obtener el título de licenciado en Bibliotecología, de igual autoría que el presente artículo. Este es orientado por las profesoras Natalia Aguirre-Ligüera y Yanet Fuster.

Nota: El editor Mario Barité aprobó este artículo.

Nota de contribución: La totalidad del trabajo estuvo a cargo de Ana Laura Gilmet Pagés.

Corrección de estilo realizada por Vanina Castellano en el marco del convenio entre la Tecnicatura universitaria en corrección de estilo (FHCE) y la Facultad de Información y Comunicación (Udelar). 